\title{
A Cluster of Major Specific Resistance Genes to Leptosphaeria maculans in Brassica napus
}

\author{
R. Delourme, M. L. Pilet-Nayel, M. Archipiano, R. Horvais, \\ X. Tanguy, T. Rouxel, H. Brun, M. Renard, and M. H. Balesdent
}

First, second, third, fourth, fifth, and eighth authors: INRA, UMR APBV, BP 35327, 35653 Le Rheu Cedex, France; sixth and ninth authors: INRA, UMR PMDV, Route de Saint-Cyr, 78026 Versailles Cedex, France; and seventh author: INRA, UMR BiO3P, BP 35327, 35653 Le Rheu Cedex, France.

Current address of M. Archipiano: Clause Tezier, Domaine de Maninet, Route de Beaumont, 26000 Valence, France.

Accepted for publication 26 January 2004.

\begin{abstract}
Delourme, R., Pilet-Nayel, M. L., Archipiano, M., Horvais, R., Tanguy, X., Rouxel, T., Brun, H., Renard, M., and Balesdent, M. H. 2004. A cluster of major specific resistance genes to Leptosphaeria maculans in Brassica napus. Phytopathology 94:578-583.

Two types of genetic resistance to Leptosphaeria maculans usually are distinguished in Brassica napus: qualitative, total resistance expressed at the seedling stage and quantitative, partial resistance expressed at the adult plant stage. The latter is under the control of many genetic factors that have been mapped through quantitative trait loci (QTL) studies using 'Darmor' resistance. The former usually is ascribed to race-specific resistance controlled by single resistance to L. maculans $(R l m)$ genes. Three B. napus-originating specific $R \operatorname{lm}$ genes $(R \operatorname{lm} 1, R \operatorname{lm} 2$, and $R \operatorname{lm} 4)$ previ-
\end{abstract}

ABSTRACT ously were characterized. Here, we report on the genetic identification of two novel resistance genes, $R \operatorname{lm} 3$ and $R \operatorname{lm} 7$, corresponding to the avirulence genes AvrLm3 and AvrLm7. The identification of a novel L. maculans-B. napus specific interaction allowed the detection of another putative new specific resistance gene, $\operatorname{Rlm} 9$. The resistance genes were mapped in two genomic regions on LG10 and LG16 linkage groups. A cluster of five resistance genes $(R \operatorname{lm} 1, R \operatorname{lm} 3, \operatorname{Rlm} 4, \operatorname{Rlm} 7$, and $R \operatorname{lm} 9)$ was strongly suggested on LG10. The relation between all these specific resistance genes and their potential role in adult-plant field resistance is discussed. These two Rlm-carrying regions do not correspond to major QTL for Darmor quantitative resistance.

Additional keywords: blackleg, Phoma lingam.
Stem canker (blackleg) caused by Leptosphaeria maculans (Desmaz.) Ces. \& De Not. (anamorph Phoma lingam) is one of the most economically important diseases of oilseed Brassica spp., especially rapeseed (Brassica napus L.), worldwide. Different sources of resistance to the pathogen have been identified and introduced into breeding lines (29). They usually are classified in two types of resistance: a qualitative resistance, expressed from the seedling stage to the adult plant stage at the cotyledon and leaf levels, which generally is ascribed to single-gene racespecific resistance (1); and a quantitative adult-stage resistance, which is controlled by many genetic factors $(24,25)$. The former type was first studied using a differential set, comprising the cvs. Westar, Glacier, and Quinta, which discriminates three pathogenicity groups (PG) (PG2 to PG4) of L. maculans (20). The four cvs. Lirabon, Glacier, Quinta, and Jet Neuf then were used to distinguish six PGs (A1 to A6) (15). Genetic approaches towards the identification of both avirulence $(A v r L m)$ genes in the pathogen and resistance $(R l m)$ genes in the plant have been developed only recently in B. napus. AvrLm1 and AvrLm2 correspond to Rlm1 and Rlm2, present in Quinta and Glacier, respectively (1). The Alml avirulence gene corresponds to the LEMI resistance gene present in Major $(10,26)$. AvrLm4 corresponds to Rlm4 present in Jet Neuf (3). More recently, novel differential interactions were identified with two Avr genes, AvrLm3 and AvrLm7, corresponding to resistance in B. napus, and three genes, AvrLm5,

Corresponding author: R. Delourme; E-mail address: rdelourm@ rennes.inra.fr

Publication no. P-2004-0406-01R

This article is in the public domain and not copyrightable. It may be freely reprinted with customary crediting of the source. The American Phytopathological Society, 2004.
AvrLm6, and AvrLm8, corresponding to L. maculans-B. juncea or $-B$. rapa specific interactions (4). To date, however, the genetic control of these novel interactions was established only on the fungus side.

With the development of molecular markers, mapping specific resistance genes has been performed in B. napus. Ferreira et al. (10) used a doubled haploid (DH) population derived from the cross Stellar $\times$ Major and mapped the gene LEMI controlling seedling resistance after inoculation with PHW1245, a "PG2" isolate harboring at least the four Avr genes AvrLm1, AvrLm2, AvrLm4, and AvrLm7 (4). DH lines also were used to map a single major locus, LmRl, controlling resistance in the Australian cv. Shiralee at the seedling stage against Western Australia isolates (19), confirming previous results obtained from Mendelian genetic analysis (33). In addition, preliminary mapping data from another Australian cultivar, Maluka, also support a single locus model for resistance and indicate that the resistance genes from Shiralee and Maluka are either linked or possibly identical (19). When analyzing common restriction fragment length polymorphism markers between $B$. napus linkage maps of Ferreira et al. (11) and Mayerhoffer et al. (19), the latter authors concluded that LmRl and LEMI mapped to the same linkage group (LG6) but at different positions. Rimmer et al. (28) compared different sources of resistance using a PG2 isolate and found that the seedling resistance gene present in Maluka could be allelic to a seedling resistance gene present in RB87-62 and linked to the major locus conferring adult-plant resistance in Crésor, previously identified by Dion et al. (8).

All of these mapping studies were performed using different, ill-defined populations of the pathogen or isolates known to harbor many different Avr genes, as is the case for PHW1245. This renders difficult the evaluation of redundancy between the puta- 
tively different target resistance genes mapped and their assignment to the $R l m$ genes genetically identified (1,3,4; this study). The recent availability of isolates of the fungus genetically bred in order to harbor as few Avr alleles as possible $(3,4,30)$ was a first step toward a better knowledge of the genomic organization of blackleg-specific resistance genes. In this study, we used such genetically bred isolates, or natural isolates fully characterized for their $A v r$ gene pattern, to map the five B. napus-originating major resistance genes (i.e., $R \operatorname{lm} 1$ to 4 and $R \operatorname{lm} 7$ ) (4). The resistance genes were mapped in two genomic regions on LG10 and LG16 of the previously published B. napus genetic map (18). A novel L. maculans $-B$. napus specific interaction was identified and was shown to involve the two same genomic regions and allowed the detection of a putative ninth specific resistance gene, $\operatorname{R} \operatorname{lm} 9$, located on LG10. The relation among all of these specific resistance genes and their potential role in adult-plant field resistance is discussed.

\section{MATERIALS AND METHODS}

Fungal materials. Fungal isolates used in this study are listed in Table 1. Field isolates PHW1245 (IBCN74) and IBCN56 were kindly provided by P. H. Williams and G. Séguin-Schwartz, respectively. Single-ascospore isolates A290 (32) and P12e were obtained from pseudothecia collected in field trials at Le Rheu (France) in 1985 and 1995, respectively. Genetically bred isolates were obtained as described in Ansan-Melayah et al. (1), Balesdent et al. (3), and Balesdent et al. (4). All isolates used were characterized for their avirulence gene pattern using a differential set comprising lines or cultivars chosen or bred to contain as few $R$ genes as possible $(3,4)$. The set comprises cv. Columbus (Rlml, $R \operatorname{lm} 3)$, cv. Bristol (Rlm2), line 22.1.1 (Rlm3), cv. Jet Neuf (Rlm4), line 23.1.1 $(R \operatorname{lm} 7)$, and cv. Westar (no $R$ gene) as a susceptible control.

Plant materials. The consensus linkage map available at INRA-Rennes is based on DH populations obtained from the three crosses Darmor- $b z h \times$ Yudal, Darmor $\times$ Samouraï, and Stellar $\times$ Drakkar (18). Except for Samouraï, none of these rapeseed lines harbors $R \operatorname{lm} 1, R \operatorname{lm} 2, R \operatorname{lm} 3, R \operatorname{lm} 4$, or $R \operatorname{lm} 7$ (Table 1). Therefore, it was necessary to set up new crosses in order to generate segregating populations for the target gene, and to check that different plant genotypes expressing the same interaction phenotype indeed possess the same $R$ gene. The different $B$. napus genotypes chosen and the segregating populations used in this study are presented in Table 1 . Quinta $\times$ Score and Glacier $\times$ Score F2 populations previously were used to study the genetic control of resistance to AvrLm1 and AvrLm2 isolates, respectively (1). A Quinta $\times$ Score backcross $(\mathrm{BC})$ population was used to study the genetic control of resistance to AvrLm4 isolates (3). In the present study, we used a DH population produced from the cross Darmor $\times$ Samourai (25), and set up a Glacier $\times$ Yudal BC population to localize Rlm2. A DH population produced from the cross Maxol $\times$ S006 was used to study Maxol-AvrLml and Maxol-AvrLm3 interactions. An F2 population derived from the cross between 23.1.1 (4) and Darmor was produced to study the 23.1.1-AvrLm7 interaction. Finally, the Darmor- $b z h \times$ Yudal DH population was used to study the Darmor- $b z h-$ IBCN56 interaction.

Resistance tests at the seedling stage. The segregating populations were tested at INRA-Versailles or INRA-Le Rheu using slightly different inoculation protocols. The DH populations were analyzed at both locations using different isolates to increase robustness and precision of the mapping. Individual plants were used for F2 and BC populations and five to seven plants were inoculated per DH line.

At INRA-Versailles, inoculations were performed as described in Balesdent et al. (3). Seed were sown in the greenhouse and cotyledons of 14-day-old seedlings were inoculated with $10-\mu 1$ droplets of inoculum $\left(10^{7}\right.$ pycnidiospores $\left./ \mathrm{ml}\right)$, with one different isolate inoculated on each half-cotyledon, so that four interactions could be assessed for every single plant. After incubation in darkness for $48 \mathrm{~h}$ at room temperature in saturated humidity, inoculated seedlings were maintained in a growth chamber at $16^{\circ} \mathrm{C}$ (night) and $24^{\circ} \mathrm{C}$ (day), with a 12 -h photoperiod. Symptoms were scored two or three times 14 to 27 days after inoculation using the 1 to 6 IMASCORE scale, where scores 1 to 3 are resistance responses (R) and scores 4 to 6 indicate susceptibility (S). In the Darmor- $b z h-$ IBCN56 interaction, $\mathrm{R}, \mathrm{S}$, or intermediate (I) phenotypes were defined based on the difference between the mean disease rating obtained with IBCN56 and the mean disease rating obtained with the virulent isolates (v11.1.1 and v23.2.1) (3) 17 days after inoculation. However, some intermediate DH lines finally were classified as S whenever IBCN56 displayed susceptibility 21 days after inoculation, or as $\mathrm{R}$ whenever the mean disease rating with IBCN56 was lower than 2. At INRA-Le Rheu, inoculations were performed as described in Williams and Delwiche (36). Seed were sown in the greenhouse and cotyledons of 10-day-old seedlings were inoculated with 10- $\mu$ d droplets of inoculum $\left(10^{7}\right.$ pycnidiospores $\left./ \mathrm{ml}\right)$. After incubation in darkness for $24 \mathrm{~h}$ at $20^{\circ} \mathrm{C}$ in saturated humidity, seedlings were maintained in a growth chamber $\left(20^{\circ} \mathrm{C}, 16\right.$-h day, 8 -h night $)$. Symptoms were scored 14 to 21 days after inoculation using a 1-to-9 scale according to lesion size, the occurrence of necrosis or chlorosis, and the presence of pycnidia (36). A qualitative scoring (resistant/susceptible) also was performed from the visual aspects of the symptoms. Resistance scoring (R) was attributed to plants showing

TABLE 1. Brassica napus segregating populations and fungal isolates used

\begin{tabular}{|c|c|c|c|c|c|c|c|}
\hline $\begin{array}{l}\text { B. napus } \\
\text { populations cross }\end{array}$ & $R$ gene $^{\mathrm{a}}$ & Type $^{\mathrm{b}}$ & No. of plants & $\begin{array}{l}\text { Isolates } \\
\text { name }\end{array}$ & Type $^{c}$ & Genotype $^{\mathrm{d}}$ & Reference \\
\hline Quinta $\times$ Score & Rlm1 Rlm4 & F2 & 110 & 11.26 .11 & Ivc & AvrLm1 avrLm2 avrLm3 avrLm4 AvrLm7 avrLm9 & 1 \\
\hline Quinta $\times$ Score & $R \operatorname{lm} 1 \boldsymbol{R} \operatorname{lm} 4$ & $\mathrm{BC}$ & 169 & $\mathrm{v} 23.2 .1$ & Ivc & avrLm1 avrLm2 avrLm3 AvrLm4 AvrLm7 avrLm9 & 4 \\
\hline Glacier $\times$ Yudal & $\operatorname{Rlm} 2 \operatorname{Rlm} 3$ & $\mathrm{BC}$ & 189 & PHW1245 & $\mathrm{F}$ & AvrLm1 AvrLm2 avrLm3 AvrLm4 AvrLm7 avrLm9 & 3,4 \\
\hline Maxol × S006 & $\operatorname{Rlm} 1 \operatorname{Rlm} 3$ & DH & 140 & $\begin{array}{c}\mathrm{P} 12 \mathrm{e} \\
\mathrm{v} 11.1 .2\end{array}$ & $\begin{array}{l}\mathrm{F} \\
\mathrm{Ivc}\end{array}$ & $\begin{array}{l}\text { AvrLm1 avrLm2 avrLm3 avrLm4 AvrLm7 avrLm9 } \\
\text { AvrLm1 avrLm2 avrLm3 avrLm4 AvrLm7 avrLm9 }\end{array}$ & $\begin{array}{c}\text { This study } \\
4\end{array}$ \\
\hline Maxol $\times$ S006 & $\operatorname{Rlm} 1 \boldsymbol{R} \operatorname{lm} 3$ & DH & 140 & 19.2.01 & Ivc & avrLm1 avrLm2 AvrLm3 avrLm4 avrLm7 avrLm9 & 4 \\
\hline 23.1.1 $\times$ Darmor & $R \operatorname{lm} 7$ & $\mathrm{~F} 2$ & 221 & A290 & $\mathrm{F}$ & avrLm1 avrLm2 avrLm3 avrLm4 AvrLm7 avrLm9 & $3,4,34$ \\
\hline Darmor- $b z h \times$ Yudal & $R \operatorname{lm} 9$ & DH & 132 & IBCN56 & $\mathrm{F}$ & AvrLm1? avrLm2? AvrLm3? avrLm4 avrLm7 AvrLm9 & This study \\
\hline
\end{tabular}


typical hypersensitive response or larger dark necrotic lesions. Susceptible scoring (S) was attributed to plants showing graygreen extending tissue collapse.

Field disease trials. The field disease trials and scoring of blackleg severity were conducted as previously described in Pilet et al. (24). The Darmor $\times$ Samourai DH population was evaluated at one location (Le Rheu, France) in 1998 and 1999 (25). The 141 $\mathrm{DH}$ lines of the Maxol $\times$ S006 population were evaluated at Le Rheu in 1999 in a randomized incomplete block design with two replicates and four blocks per replicate. Reinforced conditions of contamination were set up in the field at the three-leaf development stage by scattering rapeseed stubble collected from the previous year at Le Rheu. Blackleg severity was assessed when the plants were just beginning to ripen (June). Thirty plants per plot were uprooted and scored using the following scale based on the extent of external and internal symptoms at the crown level: $0=$ healthy plant; $1=$ external small necrotic spot; $3=$ one-sided external or small internal necrosis; $5=$ external nearly complete girdling or one-sided internal necrosis, resistant to stem bending; $7=$ deep necrosis and complete girdling, plant yellow; $9=$ broken crown, dead plant. For each plot, a mean disease index was calculated from scorings of the 30 plants uprooted using the following formula:

$$
I=\frac{1}{N} \sum_{i} i n_{i}
$$

where $N$ is the total number of scored plants, $i$ the disease score, and $n_{i}$ the number of plants with the score $i$. Adjusted mean disease index (DI) was estimated as in Pilet et al. (24), using a generalized linear model (PROC GLM, SAS/STAT software; SAS

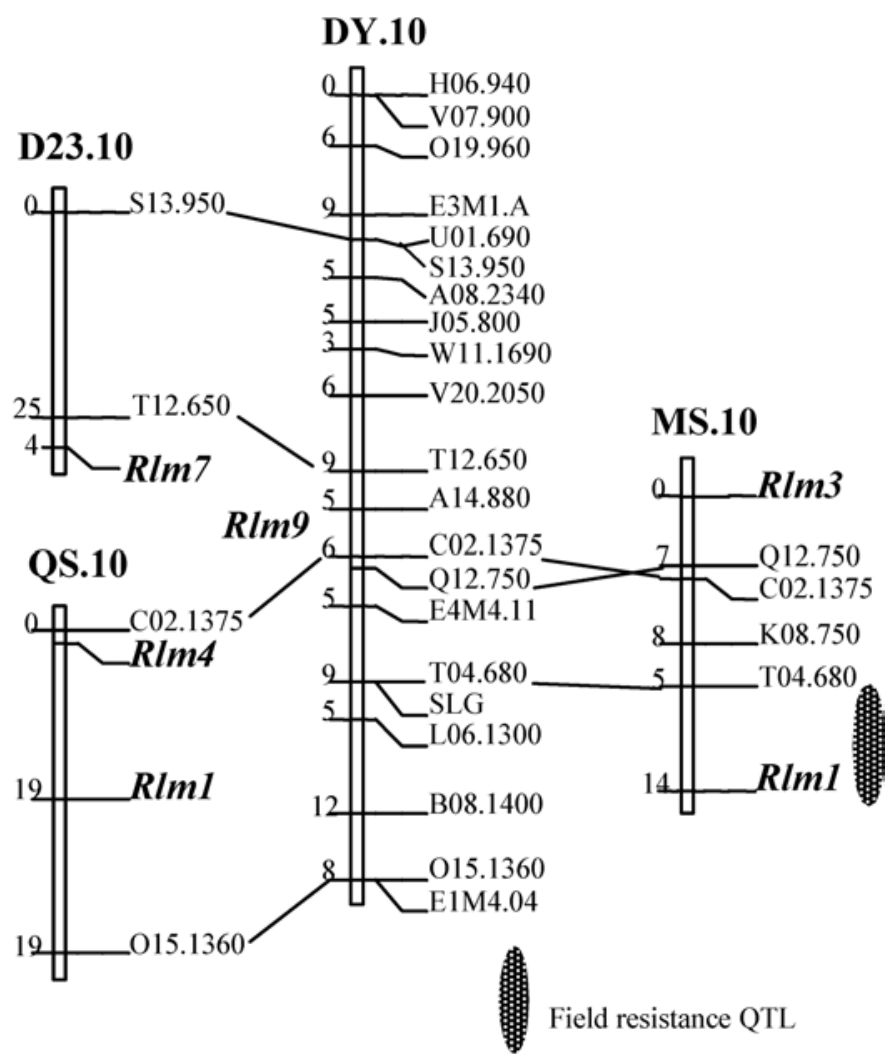

Fig. 1. Localization of $R \operatorname{lm} 1, R \operatorname{lm} 3, R \operatorname{lm} 4, R \operatorname{lm} 7$, and $R \operatorname{lm} 9$ on the linkage groups built from 23-1-1 × Darmor (D23.10), Quinta $\times$ Score (QS.10), Maxol $\times$ S006 (MS.10), and Darmor- $b z h \times$ Yudal (DY.10) crosses. The position of $\operatorname{Rlm} 9$ was deduced from a quantitative trait loci (QTL) analysis performed using quantitative data from a cotyledon test with IBCN56. The position of the QTL for mean disease index scored at the crown of the plants detected from field experiment on the Maxol $\times$ S006 doubled haploid population is indicated to the right of MS.10.
Institute, Cary, NC), which partitioned total variation into effects of lines, replicates, blocks, and errors $\left(P_{i j k}=\mu+L_{i}+R_{j}+B_{k j}+\right.$ $e_{i j k}$, where $P_{i j k}$ is the mean disease score of the $i$ th DH line located in the $k$ th block of the $j$ th replicate, $\mu$ the mean of all the data, $L_{i}$ the DH line $i$ effect, $R_{j}$ the replicate $j$ effect, $B_{k j}$ the block $k$ effect in the $j$ th replicate, and $e_{i j k}$ the residual).

Genetic mapping. Plant DNA was extracted as described in Doyle and Doyle (9). DNA bulks were made for the different segregating populations from the resistance tests data at the seedling stage. For each segregating population, DNA from eight resistant plants and eight susceptible plants were mixed into two bulks for random amplified polymorphic DNA (RAPD) marker screening through bulk segregant analysis (22). Procedure for RAPD analyses was as described in Foisset et al. (12). RAPD markers from the $B$. napus genetic maps $(12,18)$ were used in order to localize the resistance genes on the reference map. Linkage analyses were performed using MAPMAKER/EXP (version 3.0; S. E. Lincoln, M. Daly, and E. Lander, 1992. Whitehead Institute Technical Report, 3rd ed. Cambridge, MA) with a log of the likelihood ratio (LOD) score threshold of 3.0 and a recombination frequency $\leq 0.4$. Centimorgan distances were expressed with the Kosambi function (14). Quantitative trait loci (QTL) mapping was performed by composite interval mapping with the QTL Cartographer software package (version 1.21; C. J. Basten, B. S. Weir, and Z. B. Zeng, 2001. Department of Statistics, North Carolina State University, Raleigh) using a window size of 10 centimorgans $(\mathrm{cM})$ and $P$ in/out $=0.05$.

\section{RESULTS}

Rlm1 is present in Quinta and Maxol and is located on LG10. In all, 650 RAPD primers were screened on resistant or susceptible bulks made from the Quinta $\times$ Score F2 population. Twenty-two polymorphic markers discriminated the two bulks but only two markers (C02.1375 and O15.1360) were linked to RlmI (Fig. 1). These two markers previously were mapped on LG10 of the Darmor- $b z h \times$ Yudal map (18). Maxol, a cultivar genetically distant from Quinta (17), is resistant to AvrLml isolates and was suggested to harbor Rlml (30). In order to confirm the presence of Rlml in Maxol, RAPD markers from LG10 were tested for polymorphism on Maxol and S006, and a linkage group containing four markers was constructed (Fig. 1). The DH progeny was inoculated both with the field isolate P12e at Le Rheu, and with v11.1.2 at Versailles. Both experiments gave similar results; 65 lines were classified as resistant and 68 lines were classified as susceptible, which fits a $1: 1$ Mendelian ratio $\left(\chi^{2}=0.07 ; P=\right.$ $0.79)$.

The resistance gene of Maxol was mapped on the linkage group LG10 at $14.0 \mathrm{cM}$ distal to T04.680, at a position corresponding to the one obtained for Rlml in the Quinta $\times$ Score cross (Fig. 1). This confirms that the R gene present in Maxol is Rlml.

$R \operatorname{lm} 2$ is present in Glacier and Samourai and located on LG16. In all, 650 RAPD primers were screened on resistant or susceptible bulks made from the Glacier $\times$ Score F2 population. Fourteen polymorphic markers were found on the two bulks but only three markers (M08.1200, M08.600, and P02.700) were linked to $R \operatorname{lm} 2$. None of these markers was polymorphic on our $B$. napus genetic maps. Samourai recently was described as resistant to AvrLm2 isolates and suggested to harbor Rlm2 (31). In order to confirm the existence of $R \operatorname{lm} 2$ in Samouraï and map the $R$ gene, the DH population derived from the cross Darmor $\times$ Samourai was inoculated with PHW1245 at Le Rheu. In all, 56 lines were scored as resistant and 77 lines were scored as susceptible, which fits a $1: 1$ Mendelian ratio $\left(\chi^{2}=3.31 ; P=0.068\right)$. A subset of the DH lines was inoculated with 22.2.02 at Versailles and the same resistance/susceptibility response was obtained. Rlm2 was mapped on LG16 (Fig. 2) of the previously established genetic map of the Darmor $\times$ Samourai cross $(18,25) . R \operatorname{lm} 2$ then 
was mapped in the Glacier $\times$ Yudal BC population using molecular markers identified on the Glacier $\times$ Score cross and on LG16 of the B. napus genetic maps. Its position corresponds to that obtained on the Darmor $\times$ Samourai cross, therefore confirming the identity of the gene of Glacier and Samouraï (Fig. 2).

A single gene resistance to AvrLm3 isolates in Maxol is mapped on the same linkage group as $R \operatorname{lm} 1$. The DH progeny derived from the Maxol $\times$ S006 cross was used to study the genetic control of resistance of Maxol to isolate 19.2.01, which only carries AvrLm3. In all, 61 lines were scored as resistant and 56 lines as susceptible, which corresponds to a 1:1 segregation ratio $\left(\chi^{2}=0.21 ; P=0.647\right)$. This confirms the hypothesis of a single gene, $R \operatorname{lm} 3$, present in Maxol and matching AvrLm3. The segregation of $R \operatorname{lm} 3$ was compared with that of $R \operatorname{lm} 1$. A $\chi^{2}$ independence test showed that these two genes are not independent $\left(\chi^{2}=\right.$ 31.8; $P \leq 0.001)$. Rlm3 was mapped on the linkage group LG10 previously established for the localization of $R \operatorname{lm} 1$, at the other distal end compared with Rlml (Fig. 1). The genetic distance between the two genes was estimated at $34 \mathrm{cM}$ (Fig. 1).

$\boldsymbol{R} \operatorname{lm} 4$ present in Quinta is linked to $R \operatorname{lm} 1$. Following the analysis of Quinta $\times$ Score F2 and BC segregating populations, Balesdent et al. (3) suggested a genetic linkage between $R \operatorname{lm} 1$ and Rlm4. The two markers C02.1375 and O15.1360 previously identified as linked to Rlml were mapped on the Quinta $\times$ Score backcross population. $R \operatorname{lm} 4$ was mapped close to $\mathrm{C} 02.1375(3.6 \mathrm{cM})$ and the linkage between Rlml and Rlm4 was confirmed (Fig. 1).

A single gene resistance to AvrLm7 isolates is mapped on LG10. A gene-for-gene relationship was hypothesized for some B. napus lines interaction with isolates carrying AvrLm7 (4). The 23.1.1 $\times$ Darmor F2 population was used to assess this interaction using isolate A290 that carries only AvrLm7. In all, 163 plants were scored as resistant and 58 plants were scored as susceptible, which fits a $3: 1$ Mendelian ratio $\left(\chi^{2}=0.18 ; P=0.67\right)$. This confirms the hypothesis of a single gene, $\operatorname{Rlm} 7$, present in 23.1.1 line and interacting with AvrLm7. Forty RAPD markers from LG10 and LG16 were screened for polymorphism on the parental lines

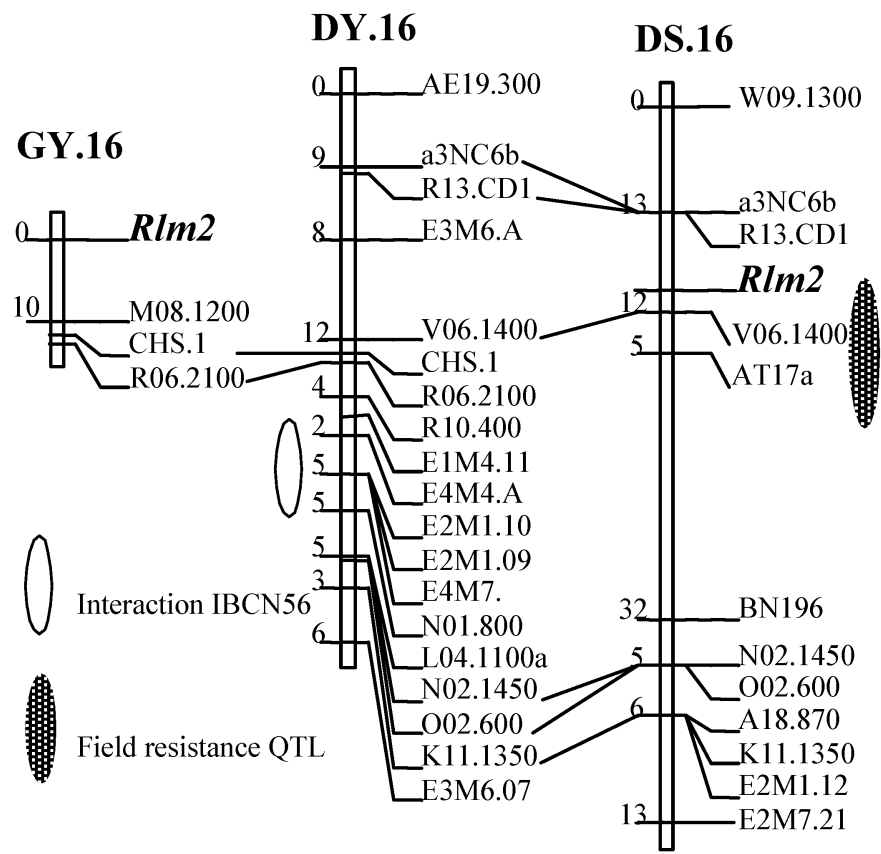

Fig. 2. Localization of $R \operatorname{lm} 2$ on the linkage groups built from Glacier $\times$ Yudal (GY.16) and Darmor $\times$ Samourai (DS.16), aligned to linkage group DY.16 from the Darmor- $b z h \times$ Yudal map. The position of the quantitative trait loci (QTL) involved in the Darmor-bzh-IBCN56 cotyledon interaction is indicated to the left of DY.16 group. The QTL to the right of the DS.16 group previously was detected from mean disease index scored at the crown of the plants from field experiments on the Darmor $\times$ Samourai doubled haploid population (25). and on two bulks of resistant or susceptible plants. Four markers were polymorphic in coupling phase with $\operatorname{Rlm} 7$ between the parental lines (S13.950 and T12.650 on LG10 and A18.870 and V06.1400 on LG16). Only T12.650 was polymorphic between the two bulks. Therefore, $R \operatorname{lm} 7$ was mapped on LG10, close to $R \operatorname{lm} 3$ and $R \operatorname{lm} 4$ (Fig. 1).

The two regions on LG10 and LG16 also are involved in the Darmor-bzh-IBCN56 interaction. A new differential interaction was identified when inoculating Darmor or Darmor- $b z h$ with the isolate IBCN56. IBCN56 was found to be avirulent on the two lines Darmor and Darmor- $b z h$, but virulent on Yudal. Therefore, the Darmor- $b z h \times$ Yudal DH progeny was used to study this interaction. A qualitative scoring was made from the scores and symptoms: 70 plants were classified as R, 20 as I, and 42 as S. If the two classes $\mathrm{R}$ and I are fused, the observed segregation fits to two segregating genes $\left(\chi^{2}=3.27 ; P=0.07\right)$. If the two classes I and $\mathrm{S}$ are fused, the observed segregation fits to one segregating gene $\left(\chi^{2}=0.48 ; P=0.49\right)$. However, the hypothesis of one major gene distinguishing the class $\mathrm{R}$ from the two others and of a second gene distinguishing the class $\mathrm{I}$ from the class $\mathrm{S}$ did not fit the observed segregation $\left(\chi^{2}=7.81 ; P=0.02\right)$. Then, the disease scores were used as quantitative data for QTL identification. Two QTL were identified: a major one on $\mathrm{LG10}\left(\mathrm{LOD}=32.9\right.$ and $R^{2}=$ $70 \%)$ and a minor one on LG16 (LOD $=7.9$ and $\left.R^{2}=9 \%\right)$. The major gene on DY10 was termed $R \operatorname{lm} 9$ and would interact with a putative AvrLm9 gene present in IBCN56. For $112 \mathrm{DH}$ lines, the genotype at Rlm 9 and at the QTL on DY16 could be deduced from their most probable positions. These results confirmed that the QTL on DY16 actually distinguished the intermediate class from the susceptible one. A significant difference (Student's $t$ test; $P=7 \times 10^{-4}$ ) between the two classes with or without the resistance allele at DY16 QTL when $\operatorname{Rlm} 9$ is present also was observed.

Rlm1 explained a great part of adult plant resistance in field conditions. A field experiment was performed at Le Rheu in 1999 on the Maxol $\times$ S006 DH population in order to evaluate the effect of Rlml on field resistance. Genotypes were distributed continuously for the resistance index but the $\mathrm{DH}$ harboring $R \operatorname{lm} 1$ were more resistant $(\mathrm{DI}=2.41)$ than the ones without $R \operatorname{lm} 1$ (DI = 4.30) (Fig. 3). QTL analysis on MS10 linkage group showed that Rlml explained $70 \%$ of the total phenotypic variation (Fig. 1).

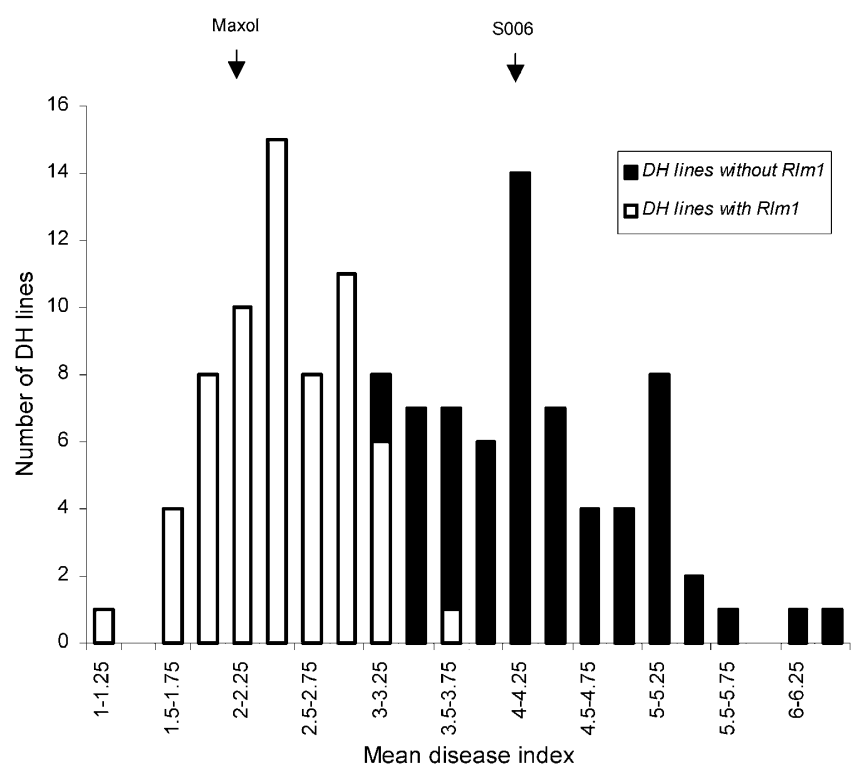

Fig. 3. Frequency distribution of the Maxol $\times$ S006 doubled haploid (DH) lines for adjusted means of the index of field resistance to Leptosphaeria maculans. The DH lines carrying Rlm1 or not carrying $R \operatorname{lm} 1$ as well as the position of parental lines are indicated. 


\section{DISCUSSION}

In this article, we report on the genetic characterization and mapping of six B. napus-originating specific resistance genes to L. maculans. A precise genetic characterization of B. napusL. maculans differential interactions was possible through the recent availability of $L$. maculans isolates genetically bred to carry as few $A v r$ genes as possible $(3,4)$. This allowed us to identify the novel resistance genes $R \operatorname{lm} 3$ and $R \operatorname{lm} 7$, corresponding to the avirulence genes AvrLm3 and AvrLm7, and to postulate a more complex novel interaction involving a major gene, $\operatorname{Rlm} 9$, and a second region with a minor effect. The genetic control of avirulence in the isolate IBCN56, which revealed the $R \operatorname{lm} 9$ gene, has yet to be established. The parallel mapping of specific resistance genes in B. napus and of Avr genes in L. maculans clearly is essential to better understand the differential interactions in this pathosystem.

Two genomic regions in B. napus were identified as carrying specific resistance genes through the study of six B. napusL. maculans interactions. One of these regions on LG16 carries $R \operatorname{lm} 2$, which was shown to be the most common $R$ gene in winter rapeseed (31). The other region on LG10 was identified in five interactions and possibly carries five specific resistance genes: $R \operatorname{lm} 1, R \operatorname{lm} 3, R \operatorname{lm} 4, R \operatorname{lm} 7$, and $R \operatorname{lm} 9$. Of these, two genes present in the Quinta lines maintained at Versailles and Rennes, Rlm 1 and $R \operatorname{lm} 4$, are linked but not allelic and two genes present in cv. Maxol, Rlm1 and Rlm3, also are linked but not allelic. Therefore, $R \operatorname{lm} 1$ is distinct from $R \operatorname{lm} 3$ and $R \operatorname{lm} 4$. Rlm 3 and $R \operatorname{lm} 4$ are found in many rapeseed cultivars but, until now, seem rarely to be present at the same time in a cultivar (M. H. Balesdent and T. Rouxel, unpublished data). Identically, and even though very few lines containing $R \operatorname{lm} 7$ were identified to date (31), $R \operatorname{lm} 7$ and $R \operatorname{lm} 3$ never were found in the same genotype (M. H. Balesdent and T. Rouxel, unpublished data). Then, the four genes Rlm3, $R \operatorname{lm} 4, R \operatorname{lm} 7$, and $R \operatorname{lm} 9$ could correspond to a cluster of tightly linked genes, to a unique gene with different alleles, or to a combination of these two hypotheses.

Interestingly, on the fungus side, an identical situation exists for the AvrLm3, AvrLm4, and AvrLm7 avirulence genes. Balesdent et al. (4) were not able to establish whether they were allelic forms of a single gene or a cluster of Avr genes. Many plant resistance genes have been described to be organized in clusters (21) and to encode intracellular-nucleotide binding leucine-rich repeat proteins with variable N-terminal domains (13). Such a clustering of $R$ genes and structural similarities leads to regions evolving more rapidly than the rest of the genome, and prone to gene duplication and intergenic recombination (27). In contrast, filamentous fungi Avr genes, even though only few of them currently are characterized, usually do not share common structural features, and may encode very diverse proteins (16). In this respect, very few clusters of $A v r$ genes have been described to date, mostly in oomycetes such as Phytophthora infestans (34) and $P$. sojae (35). Therefore, the existence of two clusters of $A v r$ genes, AvrLm1-AvrLm2-AvrLm6 and AvrLm3-AvrLm4-AvrLm7, in L. maculans was the first description of such a genome organization in filamentous fungi (4). The present study shows that there is a counterpart in the plant of the AvrLm3-AvrLm4-AvrLm7 cluster of the fungus, with three tightly linked $R$ genes, $R \operatorname{lm} 3$, $R \operatorname{lm} 4$, and $R \operatorname{lm} 7$. In contrast, $R \operatorname{lm} 1$ and $R \operatorname{lm} 2$, corresponding to the AvrLm1-AvrLm2-AvrLm6 cluster, are unlinked and located on different linkage groups. To our knowledge, such a cluster-tocluster relationship has never been described in plant-pathogen interactions, and it offers fascinating perspectives to understand the functional and evolutionary relationships of these genes on the fungal and the plant side. One of the hypotheses to assess would be whether the $A v r$ genes or alleles encode closely related products that are recognized by closely related $R$ genes that evolved through gene duplication.
Our results are difficult to relate to previously published mapping studies of $R$ genes in B. napus because of the unknown Avr composition of most of the isolates used and because of the absence of common segregating markers. Ferreira et al. (10) mapped LEMI from Major using isolate PHW1245. Major recently has been shown to possess $R \operatorname{lm} 4$, and to be devoid of Rlm 1 , $R \operatorname{lm} 2$, and $R \operatorname{lm} 7$, but it was not challenged with $A v r L m 3$ or AvrLm9 isolates in the study by Rouxel et al. (31). However, because PHW1245 does not carry AvrLm3 or AvrLm9, it seems more likely that $L E M 1$ corresponds to Rlm4. LEM1 was located on the same linkage group as the resistance genes described in Crésor $(8,28)$ or in Australian cvs. Maluka and Shiralee $(19,28)$. The mapping performed here indicates that all these studies were targeted at a linkage group corresponding to LG10. Furthermore, because Maluka also only harbors Rlm4, which is the most common R gene in Australian spring rapeseed (31), it can be postulated that most or the entire mapping previously performed was targeted at $R \operatorname{lm} 4$.

The efficiency of some of the L. maculans specific resistance genes was assessed in the field. Rlml present in Maxol was shown to be efficient in the field because it explained $70 \%$ of the variation for resistance at the adult plant stage. The frequency of the AvrLml gene in the L. maculans population present in the field is not precisely known. It was assessed in autumn 1995 and 1999 on a set of 36 isolates per year and was estimated at $90 \%$ (23) and 50\% (30), respectively. The field experiment on Maxol $\times$ S006 DH populations was performed in 1998-99, at a time when the frequency of AvrLml could be estimated to be close to $50 \%$. The frequency of AvrLm3 was not assessed but it has been found to be very low in L. maculans isolates originating from different part of France (M. H. Balesdent and T. Rouxel, unpublished data). Moreover, the position of the QTL on MS10 corresponds to the Rlm I position. Therefore, the adult resistance in Maxol is explained mainly by the presence of $R \operatorname{lml}$, which is efficient when isolates harboring AvrLml are prevalent or an important part of the pathogen population $(2,30)$. The position of $R \operatorname{lm} 2$ on LG16 corresponds to a QTL identified for adult plant resistance in the Darmor $\times$ Samourai DH population (25). Samourai carries the resistance allele at this QTL as well as $R \operatorname{lm} 2$. No French isolates carry AvrLm2 (30); therefore, two hypotheses can be proposed to explain this colocalization: either the $R \operatorname{lm} 2$ gene has a residual effect at the adult plant stage, as has been suggested in other pathosystems (7), or genes linked to Rlm2 are responsible for part of the variation for resistance at this QTL. The position of this QTL for adult plant resistance also could correspond to one of the QTL involved in Darmor-bzh-IBCN56 interaction. The precision of the mapping has to be improved, but this could indicate that a genetic factor that induces slower resistance reactions and leads to intermediate resistance response at the cotyledon stage also could be involved in adult plant resistance.

The two regions on LG10 and LG16 identified as carrying specific resistance genes to $L$. maculans do not correspond to the genomic regions carrying the most consistent QTL involved in the quantitative adult plant resistance present in Darmor $(24,25)$. Neither do they correspond to the regions where interspecific resistance genes were introgressed from $B$. juncea (5) or from $B$. nigra (A. M. Chèvre, unpublished data). From all these data, breeding schemes aiming at associating intra- or interspecific major resistance genes or QTL within a genotype or in varietal associations can be proposed. The choice of the strategy will depend on the efficiency of the constructed resistance and on its durability. It already is known that $L$. maculans has a very high potential to break down major resistance genes and that single specific resistance genes do not provide a durable resistance. This was shown in an experimental design for the Jlm1/Rlm6 gene introgressed from $B$. juncea (6) and in real cropping conditions for the Rlml cultivars (30). Extensive studies on the durability of various resistance genetic factors combinations, on the relative fitness of some 
$a v r / A v r$ genes, on the effect of the plant genetic background and cultural techniques are currently in progress in order to propose an efficient management of all of these resistance factors.

\section{ACKNOWLEDGMENTS}

This work was partly supported by a grant from the Société Interprofessionnelle Des Oléagineux, protéagineux et cultures textiles (SIDO) and the Agence De l'Environnement et de la Maîtrise de l'Energie (ADEME). We thank A. M. Chèvre and M. Manzanares-Dauleux for critical reading of the manuscript.

\section{LITERATURE CITED}

1. Ansan-Melayah, D., Balesdent, M. H., Delourme, R., Pilet, M. L., Tanguy, X., Renard, M., and Rouxel, T. 1998. Genes for race-specific resistance against blackleg disease in Brassica napus L. Plant Breed. 117:373-378.

2. Ansan-Melayah, D., Rouxel, T., Bertrandy, J., Letarnec, B., MendesPereira, E., and Balesdent, M. H. 1997. Field efficiency of Brassica napus specific resistance correlates with Leptosphaeria maculans population structure. Eur. J. Plant Pathol. 103:835-841.

3. Balesdent, M. H., Attard, A., Ansan-Melayah, D., Delourme, R., Renard, M., and Rouxel, T. 2001. Genetic control and host range of avirulence toward Brassica napus cultivars Quinta and Jet Neuf in Leptosphaeria maculans. Phytopathology 91:70-76.

4. Balesdent, M. H., Attard, A., Kühn, M. L., and Rouxel, T. 2002. New avirulence genes in the phytopathogenic fungus Leptosphaeria maculans. Phytopathology 92:1122-1133.

5. Barret, P., Guérif, J., Reynoird, J. P., Delourme, R., Eber, F., Renard, M., and Chèvre, A. M. 1998. Selection of stable Brassica napus-Brassica juncea recombinant lines resistant to blackleg (Leptosphaeria maculans). 2. A "to and fro" strategy to localize and characterize interspecific introgressions on the B. napus genome. Theor. Appl. Genet. 96:1097-1103.

6. Brun, H., Levivier, S., Ruer, D., Somda, I., Chèvre, A. M., and Renard, M. 2000. A field method for evaluating the potential durability of new resistance sources: Application to the Leptosphaeria maculans/Brassica napus pathosystem. Phytopathology 90:961-966.

7. Chantret, N., Pavoine, M. T., and Doussinault, G. 1999. The race-specific resistance gene to powdery mildew ' $M I R E$ ' has a residual effect on adult plant resistance of winter wheat line RE714. Phytopathology 89:533-539.

8. Dion, Y., Landry, B. S., Gugel, R. K., and Seguin-Swartz, G. 1995. RFLP mapping of blackleg resistance on the canola genome. Blackleg News 2:1-3.

9. Doyle, J. J., and Doyle, J. L. 1990. Isolation of plant DNA from fresh tissue. Focus 12:13-15.

10. Ferreira, M. E., Rimmer, S. R., Williams, P. H., and Osborn, T. C. 1995. Mapping loci controlling Brassica napus resistance to Leptosphaeria maculans under different screening conditions. Phytopathology 85:213217.

11. Ferreira, M. E., Williams, P. H., and Osborn, T. C. 1994. RFLP mapping of Brassica napus using doubled haploid lines. Theor. Appl. Genet. 89:615-621.

12. Foisset, N., Delourme, R., Barret, P., Hubert, N., Landry, B. S., and Renard, M. 1996. Molecular mapping analysis in Brassica napus using isozyme, RAPD and RFLP markers on a doubled haploid progeny. Theor. Appl. Genet. 93:1017-1025.

13. Hammond-Kosack, K. E., and Parker, J. E. 2003. Deciphering plantpathogen communication: Fresh perspectives for molecular resistance breeding. Curr. Opin. Biotechnol. 14:177-193.

14. Kosambi, D. D. 1944. The estimation of map distances from recombination values. Ann. Eugen. 12:172-175.

15. Kuswinanti, T., Sock, J., and Hoppe, H. H. 1995. Variation of aggressive isolates of Leptosphaeria maculans based on cotyledon reactions on extended differential set. Pages 1248-1250 in: Proc. 9th Int. Rapeseed Congr., Cambridge, UK.

16. Laugé, R., and De Wit, P. J. G. M. 1998. Fungal avirulence genes: Structure and possible functions. Fungal Genet. Biol. 24:285-297.
17. Lombard, V., Baril, C. P., Dubreuil, P., Blouet, F., and Zhang, D. 2000. Genetic relationships and fingerprinting of rapeseed cultivars by AFLP: Consequences for varietal registration. Crop Sci. 40:1417-1425.

18. Lombard, V., and Delourme, R. 2001. A consensus linkage map for rapeseed (Brassica napus L.): Construction and integration of three individual maps from DH populations. Theor. Appl. Genet. 103:491-507.

19. Mayerhofer, R., Bansal, V. K., Thiagarajah, G. R., Stringam, G. R., and Good, A. G. 1997. Molecular mapping of resistance to Leptosphaeria maculans in Australian cultivars of Brassica napus. Genome 40:294-301.

20. Mengistu, A., Rimmer, S. R., Koch, E., and Williams, P. H. 1991. Pathogenicity grouping of isolates of Leptosphaeria maculans on Brassica napus cultivars and their disease reaction profiles on rapid-cycling Brassicas. Plant Dis. 75:1279-1282.

21. Michelmore, R. W., and Meyers, B. C. 1998. Clusters of resistance genes in plants evolve by divergent selection and a birth-and-death process. Genome Res. 8:1113-1130.

22. Michelmore, R. W., Paran, I., and Kesseli, R. V. 1991. Identification of markers linked to disease-resistance genes by bulked segregant analysis: A rapid method to detect markers in specific genomic regions by using segregating populations. Proc. Natl. Acad. Sci. USA 88:9828-9832.

23. Pilet, M. L. 1999. Analyse génétique de la résistance du colza à la nécrose du collet et à la cylindrosporiose à l'aide de marqueurs moléculaires. $\mathrm{PhD}$ thesis. Institut National Agronomique, Paris-Grignon.

24. Pilet, M. L., Delourme, R., Foisset, N., and Renard, M. 1998. Identification of loci contributing to quantitative field resistance to blackleg disease, causal agent Leptosphaeria maculans (Desm.) Ces. et de Not., in winter rapeseed (Brassica napus L.). Theor. Appl. Genet. 96:23-30.

25. Pilet, M. L., Duplan, G., Archipiano, M., Barret, P., Baron, C., Horvais, R., Tanguy, X., Lucas, M., Renard, M., and Delourme, R. 2001. Stability of QTL for field resistance to blackleg across two genetic backgrounds in oilseed rape. Crop Sci. 41:197-205.

26. Pongam, P., Osborn, T. C., and Williams, P. H. 1998. Genetic analysis and identification of amplified fragment length polymorphism markers linked to the alm1 avirulence gene of Leptosphaeria maculans. Phytopathology 88:1068-1072.

27. Richter, T. E., and Ronald, P. C. 2000. The evolution of disease resistance genes. Plant Mol. Biol. 42:195-204.

28. Rimmer, S. R., Borhan, M. H., Zhu, B., and Somers, D. 1999. Mapping resistance genes in Brassica napus to Leptosphaeria maculans. Proc. 10th Int. Rapeseed Congr. Canberra, Australia.

29. Rimmer, S. R., and Van Den Berg, C. G. J. 1992. Resistance of oilseed Brassica spp. to blackleg caused by Leptosphaeria maculans. Can. J. Plant Pathol. 14:56-66.

30. Rouxel, T., Penaud, A., Pinochet, X., Brun, H., Gout, L., Delourme, R., Schmidt, J., and Balesdent, M. H. A ten-year survey of populations of Leptosphaeria maculans in France indicates a rapid adaptation towards the Rlm1 resistance gene of oilseed rape. Eur. J. Plant Pathol. 109:871881.

31. Rouxel, T., Willner, E., Coudard, L., and Balesdent, M. H. 2003. Screening and identification of resistance to Leptosphaeria maculans (stem canker) in Brassica napus accessions. Euphytica 133:219-231.

32. Somda, I., Delourme, R., Renard, M., and Brun, H. 1999. Pathogenicity of Leptosphaeria maculans isolates on a Brassica napus-B. juncea recombinant line. Phytopathology 89:169-175.

33. Stringam, G. R., Bansal, V. K., Thiagarajah, M. R., and Tewari, J. P. 1992. Genetic analysis of blackleg (Leptosphaeria maculans) resistance in Brassica napus L. using the doubled haploid method. Pages 213-214 in: Thirteenth Eucarpia Congr., Angers, France.

34. Van der Lee, T., Testa, A., van't Klooster, J., van den Berg-Velthuis, G., and Govers, F. 2001. Chromosomal deletion in isolates of Phytophthora infestans correlates with virulence on R3, R10 and R11 potato lines. Mol. Plant-Microbe Interact. 14:1444-1452.

35. Whisson, S. C., Drenth, A., Maclean, D. J., and Irwin, J. A. G. 1995. Phytophthora sojae avirulence genes, RAPD and RFLP markers used to construct a detailed genetic linkage map. Mol. Plant-Microbe Interact. 8:988-995.

36. Williams, P. H., and Delwiche, P. A. 1979. Screening for resistance to blackleg of Crucifers in the seedling stage. Pages 164-170 in: Proc. Eucarpia Conf. Breed. Cruciferous Crops, Wageningen, Pays-Bas, the Netherlands. 